\title{
New Statistical Multiparticle Approach to the Acceleration of Electrons by the Ion Field in Plasmas
}

\author{
Eugene Oks \\ Department of Physics, Auburn University, 206 Allison Laboratory, Auburn, AL 36849, USA \\ Correspondence should be addressed to Eugene Oks, goks@physics.auburn.edu
}

Received 30 April 2009; Accepted 8 June 2009

Academic Editor: Elisabeth Dalimier

Copyright () 2010 Eugene Oks. This is an open access article distributed under the Creative Commons Attribution License, which permits unrestricted use, distribution, and reproduction in any medium, provided the original work is properly cited.

The phenomenon of the acceleration of the (perturbing) electrons by the ion field (AEIF) significantly reduces Stark widths and shifts in plasmas of relatively high densities and/or relatively low temperature. Our previous analytical calculations of the AEIF were based on the dynamical treatment: the starting point was the ion-microfield-caused changes of the trajectories and velocities of individual perturbing electrons. In the current paper, we employ a statistical approach: the starting point is the electron velocity distribution function modified by the ion microfield. The latter had been calculated by Romanovsky and Ebeling in the multiparticle description of the ion microfield. The result shows again the reduction of the electron Stark broadening. Thus two totally different analytical approaches (dynamical and statistical) agree with each other and therefore disprove the corresponding recent fully-numerical simulations by Stambulchik et al. that claimed an increase of the electron Stark broadening.

\section{Introduction}

The phenomenon of the acceleration of the (perturbing) electrons by the ion field (AEIF) significantly reduces Stark widths and shifts in plasmas of relatively high densities and/or relatively low temperature. This phenomenon was first described analytically in the binary approach in paper [1] with subsequent analytical improvements in paper [2]. Then it was also described analytically in the multiparticle approach in book [3] and paper [4]. The essence of this phenomenon is the following.

Due to the presence of the ion field, perturbing electrons are passing by the radiator at higher velocities than it would be at the absence of the ion field. The increase of the electron velocities translates into a decrease of the electron broadening (i.e., a decrease of Stark widths and shifts). The narrowing (and shift-reducing) phenomenon caused by the AEIF is the realization of a direct coupling between electrons and ions. It affects the spectral line shape in addition to the effect of the indirect coupling between the electron and ion broadenings (see, e.g., [3]). The latter coupling is indirect because it is carried out via the radiating atom which acts as an intermediary, this being manifested by the fact that the coupling parameter depends on the quantum numbers of the atomic states (as well as on the parameters of the electron and ion microfields). In distinction to this, the coupling parameter in the narrowing phenomenon does not depend on the atomic quantum numbers. It should be emphasized that in the conventional theory [5] there was no coupling of any kind between the electrons and ions.

The analytically described direct and indirect couplings of the electron and ion microfields combined with the analytical description of the ion dynamics constituted a highly advanced theory of the Stark broadening $[3,4]$. A code based on this analytical theory eliminated significant discrepancies between variety of benchmark experiments and previous theories and/or simulations $[3,4]$.

However, there are two different schools of thought on what should be the ultimate test of various theories. One school of thought considers the comparison with benchmark experiments as the ultimate test of the theory. For spectral line shapes in plasmas, benchmark experiments are those, where plasma parameters are determined independently of the spectral line shape theory to be tested.

Another school of thought insists that the ultimate test of a particular theory is the comparison with results of a code based on fully-numerical simulations starting from the "scratch" rather than from some analytical advance. 
Within this school of thought, there have been recently conducted fully numerical simulations trying to "mimic" the phenomenon of AEIF [6]. Based on their fully-numerical simulations conducted for the $H_{\alpha}$ line at just one value of the electron density $N_{e}$ and just one value of the temperature $T$, the authors of [6] claimed that the AEIF leads to an increase of the electron-caused Stark width rather than to its decrease.

It should be emphasized that those simulations [6] had lots of limitations. The primary limitation was their employment of the binary version of the AEIF. Thus, their results have no bearing on the analytical results for the AEIF obtained in the multiparticle approach $[3,4]$. Nevertheless, the controversial results of simulation from [6] for the binary version of the AEIF required a resolution.

In the current paper we resolve this issue as follows. Our previous analytical calculations of the AEIF [1-4] were based on the dynamical treatment of the perturbing electrons. In other words, in [1-4] we calculated analytically how the ion microfield changes the trajectories and velocities of the individual perturbing electrons and then averaged their contribution to the broadening over the ensemble of electrons. In the current paper, instead of the dynamical treatment we employ a statistical approach. Namely, we start from the electron velocity distribution function modified by the presence of the ion microfield-this modified electron velocity distribution function had been calculated (for a different purpose) by Romanovsky and Ebeling in the multiparticle description of the ion microfield [7]. With the help of the modified electron velocity distribution function from [7], we then calculate the Stark broadening by electrons within the framework of the conventional theory usually assigned to Griem [5] (who is one of the coauthors of [6]). The result shows that the electron Stark broadening decreases.

Thus two totally different analytical approaches (dynamical and statistical) agree with each other (by predicting a decrease of the electron Stark broadening) and therefore disprove the fully-numerical simulations from [6] (that claimed an increase of the electron Stark broadening). In conclusion we briefly discuss possible reasons for the failure of the fully-numerical simulations from [6].

\section{Analytical Calculations}

Romanovsky and Ebeling [7] considered the instantaneous state of a plasma as a set as "domains" (the size of the domains being of the order of the Debye radius) with different constant values of the ion microfield $\mathbf{F}$. The latter was treated in the multiparticle description. The characteristic time of the domain structure changes is of the order of the inverse plasma frequency. By applying the statistical approach, Romanovsky and Ebeling derived the following (unnormalized) velocity distribution function of the plasma electrons affected by the ion microfield

$$
f^{(u)}(v)=\left(\frac{2 v}{\pi}\right) \int_{0}^{\infty} d t \sin (t v) \exp \left[-\left(v_{E} t\right)^{3 / 2}-\frac{\left(v_{T} t\right)^{2}}{4}\right],
$$

where the superscript " $u$ " stands for "un-normalized", $v_{T}=$ $\left(2 T_{e} / m\right)^{1 / 2}$ is the mean thermal velocity of the electrons, and $v_{F}$ is the scaled (to the dimension of velocity) characteristic ion microfield defined as follows:

$$
v_{E}=\left(\frac{e E_{H}}{m}\right)\left[\frac{m}{4 \pi e^{2} N_{i}}\right]^{1 / 2}(T i / T e)^{1 / 2} .
$$

Here $e, m$, and $T_{e}$ are the electron charge, mass, and temperature, respectively; $N_{i}$ and $T_{i}$ are ion density and temperature, respectively; the quantity

$$
E_{H}=2 \pi\left(\frac{4}{15}\right)^{2 / 3} e N_{i}^{2 / 3}
$$

is the characteristic Holtsmark microfield. ( The authors of [7] used $F_{H}$ in the form $E_{H}=2 \pi(4 / 15)^{2 / 3} e\left(N_{i}^{2 / 3}+N_{e}^{2 / 3}\right)$, where $N_{e}$ was the electron density-because the focus of their study was the effect of the plasma microfield on the ionization by very rapid electrons having the kinetic energy much greater than $T_{e}$. Those electrons were affected by both the ion and electron microfields. ) The distribution function $f(v)$ becomes the Maxwell distribution if $v_{E}=0$, and it becomes the Holtsmark distribution if $v_{T}=0$.

Let us first consider the most practically important case where $v_{E} \ll v_{T}$. In this case we can expand $\exp \left[-\left(v_{F} t\right)^{3 / 2}\right]$ in the integrand in (1):

$$
\begin{aligned}
f_{\text {small }}^{(u)}(\mathrm{v})= & \left(\frac{2 v}{\pi}\right) \int_{0}^{\infty} d t \sin (t v) \\
& \times\left[1-\left(v_{E} t\right)^{3 / 2}\right] \exp \left[-\frac{\left(v_{T} t\right)^{2}}{4}\right] .
\end{aligned}
$$

Here subscript "small" stands for a relatively small ion density/field. After calculating the integral in (4) analytically and then normalizing the corresponding distribution function (also analytically), we obtain the following normalized velocity distribution function:

$$
\begin{aligned}
f_{\text {small }}(v)= & {\left[\frac{4 v^{2}}{\pi v_{T}^{9 / 2}}\right] } \\
\times & {\left[\pi^{1 / 2} v_{T}^{3 / 2} \exp \left(-\frac{v^{2}}{v_{T}^{2}}\right)\right.} \\
& \left.\quad-2^{5 / 2} \Gamma\left(\frac{9}{4}\right) v_{E}^{3 / 2} F\left(\frac{9}{4}, \frac{3}{2} ;-\frac{v^{2}}{v_{T}^{2}}\right)\right] .
\end{aligned}
$$

Here $\Gamma(z)$ is the gamma-function, $F(a, c ; z)$ is the confluent hypergeometric function.

At the stage preceding the averaging over velocities, the electron impact broadening operator in the dipole approximation has the following form (in accordance to the conventional theory [5]):

$$
\Phi(v)=\frac{K}{v^{2}},
$$

where $K$ is a well-known operator that practically does not depend on velocity (see, e.g., [8])

$$
K=-\left(\mathbf{r}_{a} \mathbf{r}_{a}-2 \mathbf{r}_{a} \mathbf{r}_{b}^{*}+\mathbf{r}_{b}^{*} \mathbf{r}_{b}^{*}\right)\left[\frac{4 \pi e^{4} N_{e}}{3 \hbar^{2}}\right]\left[\ln \left(\frac{\rho_{\max }}{\rho_{\min }}\right)+\frac{1}{2}\right] .
$$




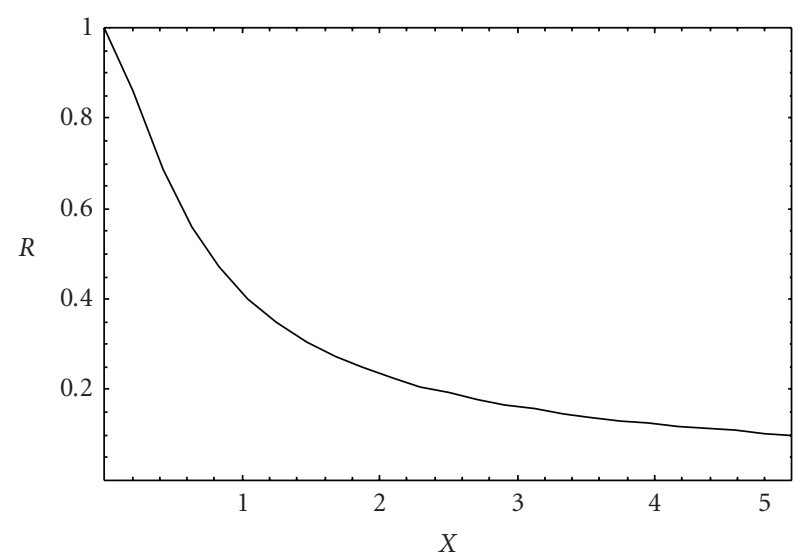

Figure 1: The electron broadening reducing factor $R$ versus the parameter $X=v_{E} / v_{T}$ representing the scaled dimensionless ion density/field. Here $v_{T}=\left(2 T_{e} / m\right)^{1 / 2}$, and $v_{E}$ is defined by (2), (3).

The next step is the usual averaging over velocities

$$
\Phi_{\text {small }}=\int_{0}^{\infty} d v v f_{\text {small }}(v) \Phi(v)
$$

By calculating the integral in (8) analytically, we obtained

$$
\Phi_{\text {small }}=\left[\frac{2 K}{\pi^{1 / 2} v_{T}}\right]\left[1-\frac{2^{3 / 2} \Gamma(5 / 4) v_{E}^{3 / 2}}{\left(\pi^{1 / 2} v_{T}^{3 / 2}\right)}\right] .
$$

From (9) it is clearly seen that as the parameter $v_{E} / v_{T}$ (representing the scaled dimensionless ion density/field) increases from zero, the electron impact broadening decreases. Just this result alone disproves the claim by the authors of [6] that the AEIF leads to an increase of the electron-caused Stark width.

For completeness, let us now consider the opposite case: $v_{E} \gg v_{T}$. In this case we can expand $\exp \left[-\left(v_{T} t\right)^{2} / 4\right]$ in the integrand in (1):

$$
\begin{aligned}
f_{\text {large }}^{(u)}(\mathrm{v})= & \left(\frac{2 v}{\pi}\right) \int_{0}^{\infty} d t \sin (t v) \\
& \times\left[1-\frac{\left(v_{T} t\right)^{2}}{4}\right] \exp \left[-\left(v_{E} t\right)^{3 / 2}\right] .
\end{aligned}
$$

Here subscript "large" stands for a relatively large ion density/field. After calculating the integral in (10) analytically and then normalizing the corresponding distribution function (also analytically), we obtain the normalized velocity distribution function $f_{\text {large }}(v)$. The expression for $f_{\text {large }}(v)$ is rather bulky, and we omit it (since it is only an intermediate result). Then by using $f_{\text {large }}(v)$, we perform the usual averaging over velocities

$$
\Phi_{\text {large }}=\int_{0}^{\infty} d v v f_{\text {large }}(v) \Phi(v)
$$

By calculating the integral in (11) analytically, we obtained the following relatively simple result:

$$
\Phi_{\text {large }}=\left[\frac{9 K \Gamma(11 / 3)}{20 \pi v_{E}}\right]\left[1-\frac{20 v_{T}^{2}}{27 \Gamma(11 / 3) v_{E}^{2}}\right]
$$

To better visualize our results, we define in a natural way the electron broadening reducing factor

$$
R=\frac{\Phi\left(v_{E} / v_{T}\right)}{\Phi(0)}
$$

Figure 1 shows the dependence of the electron broadening reducing factor $R$ on the parameter $X=v_{E} / v_{T}$ representing the scaled dimensionless ion density/field. It is seen that the new statistical approach used in this paper confirms that the effect of the ion microfield on the electron broadening is the reduction of the electron Stark widths and shifts.

\section{Conclusions}

We performed analytical calculations of the phenomenon of AEIF based on the new statistical multiparticle approach originating from Romanovsky-Ebeling's paper [7]. We showed that the results are in agreement with the previous analytical results obtained by a different (dynamical rather than statistical) approach employed in [1-4]. Both approaches demonstrated a decrease of the electron Stark broadening. This is a clear indication that the results of fully-numerical simulations [6] claiming an increase of the electron Stark broadening are incorrect.

The failure of the fully-numerical simulations from [6] could be due to quite general reasons. Complicated codes (such as the one from [6]) require adequate verification and adequate validation [9]. The verification is the determination that the code solves the chosen model correctly. Validation is the determination that the model itself captures the essential physical phenomena with adequate fidelity. Post and Votta [9] noted that without adequate verification and validation, computational results are not credible.

Complicated codes are a collection of individual blocks or components. Even if the individual blocks are relatively accurate, the entire code may be not. In another publication [10] Post and Votta emphasized that since a computational simulation is only a model of nature, not nature itself, there is no assurance that a collection of accurate individual components will capture the emergent effects.

There are numerous failures of complicated large-scale codes/simulations (see, e.g., [9]). In the area of plasma fusion-the area for which spectral line shapes serve as diagnostics-examples are the following [9]. By stretching boundary conditions far beyond what could be scientifically justified, computer simulations were able to "reproduce" the exciting but wrong experimental discovery of sonoluminescent fusion. With regard to the International Thermonuclear Experimental Reactor (ITER), preliminary computational predictions in 1996 of inadequate performance by the proposed facility were wrongly characterized as definitive. Those predictions contributed to the 1998 US withdrawal from that important and promising international undertaking.

So, the failure of the fully-numerical simulations from [6] probably resulted from inadequate verification and/or inadequate validation. By the way, the authors of [6] admit that their simulations failed to reproduce straightforward analytical calculations from [11] concerning the breakdown 
of the so-called "line space concept," while they did not find any intrinsic deficiency of those analytical calculations. (The line space concept limits the lineshape calculations to the direct product of the two manifolds, corresponding to the upper and lower principal quantum numbers involved in the radiative transition.) This failure of the authors of [6] should have warned them about seemingly inadequate verification and/or inadequate validation of their code.

The last but not least: other codes (developed by Alexiou, who is one of the coauthors of [6]), that are similar in nature to the one from [6], fail to agree with the benchmark experiments by about 30\%-as described in detail in [3]. This discrepancy with the benchmark experiments is by one order of magnitude higher than the estimate of the code inaccuracy provided in [6], so that the latter seems to be significantly underestimated.

\section{References}

[1] E. Oks, "A new spectroscopic effect resulting in a narrowing of hydrogen lines in dense plasmas," Journal of Quantitative Spectroscopy and Radiative Transfer, vol. 65, no. 1-3, pp. 405414, 2000.

[2] E. Oks, "Reduction of spectral line shifts due to the acceleration of electrons by the ion field in plasmas," Journal of Physics B, vol. 35, no. 10, pp. 2251-2260, 2002.

[3] E. Oks, Stark Broadening of Hydrogen and Hydrogenlike Spectral Lines in Plasmas: The Physical Insight, Alpha Science International, Oxford, UK, 2006.

[4] E. Oks, "Stark Widths of Hydrogen Spectral Lines in Plasmas: a Highly-Advanced Non-Simulative Semiclassical Theory and Tables," in Spectral Line Shapes, E. Oks and M. Pindzola, Eds., vol. 18 of AIP Conference Proceedings no. 874, p. 19, AIP Press, New York, NY, USA, 2006.

[5] H. R. Griem, Spectral Line Broadening by Plasmas, Academic Press, New York, NY, USA, 1974.

[6] E. Stambulchik, D. V. Fisher, Y. Maron, H. R. Griem, and S. Alexiou, "Correlation effects and their influence on line broadening in plasmas: application to $\mathrm{H}_{\alpha}$," High Energy Density Physics, vol. 3, no. 1-2, pp. 272-277, 2007.

[7] M. Yu. Romanovsky and W. Ebeling, "Corrections of electron impact ionization rates by plasmas electric microfield," Physics Letters A, vol. 317, no. 1-2, pp. 150-155, 2003.

[8] V. S. Lisita, "Stark Broadening of Hydrogen Lines in Plasmas," Soviet Physics Uspekhi, vol. 122, p. 449, 1977.

[9] D. E. Post and L. G. Votta, "Computational science demands a new paradigm," Physics Today, vol. 58, no. 1, pp. 35-41, 2005.

[10] Physics Today, p. 14, August 2005.

[11] R. W. Lee and E. Oks, "Breakdown of the line-space concept in Stark broadening of spectral lines by plasmas," Physical Review E, vol. 58, no. 2, pp. 2441-2445, 1998. 


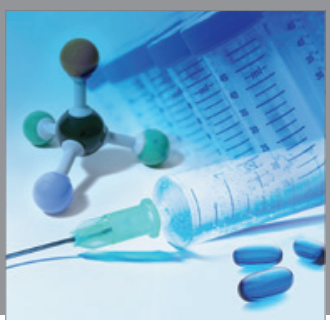

International Journal of

Medicinal Chemistry

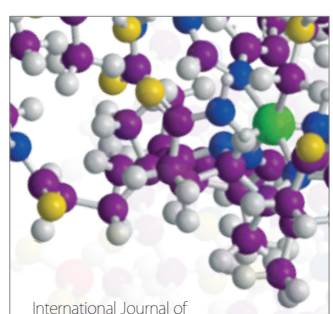

Carbohydrate Chemistry

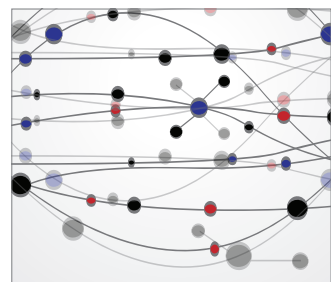

The Scientific World Journal
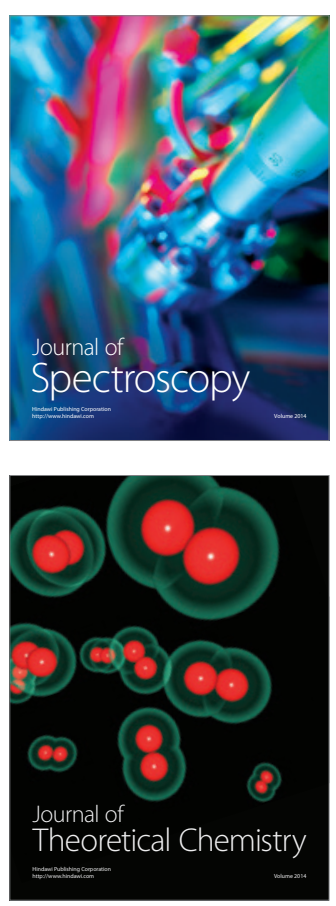
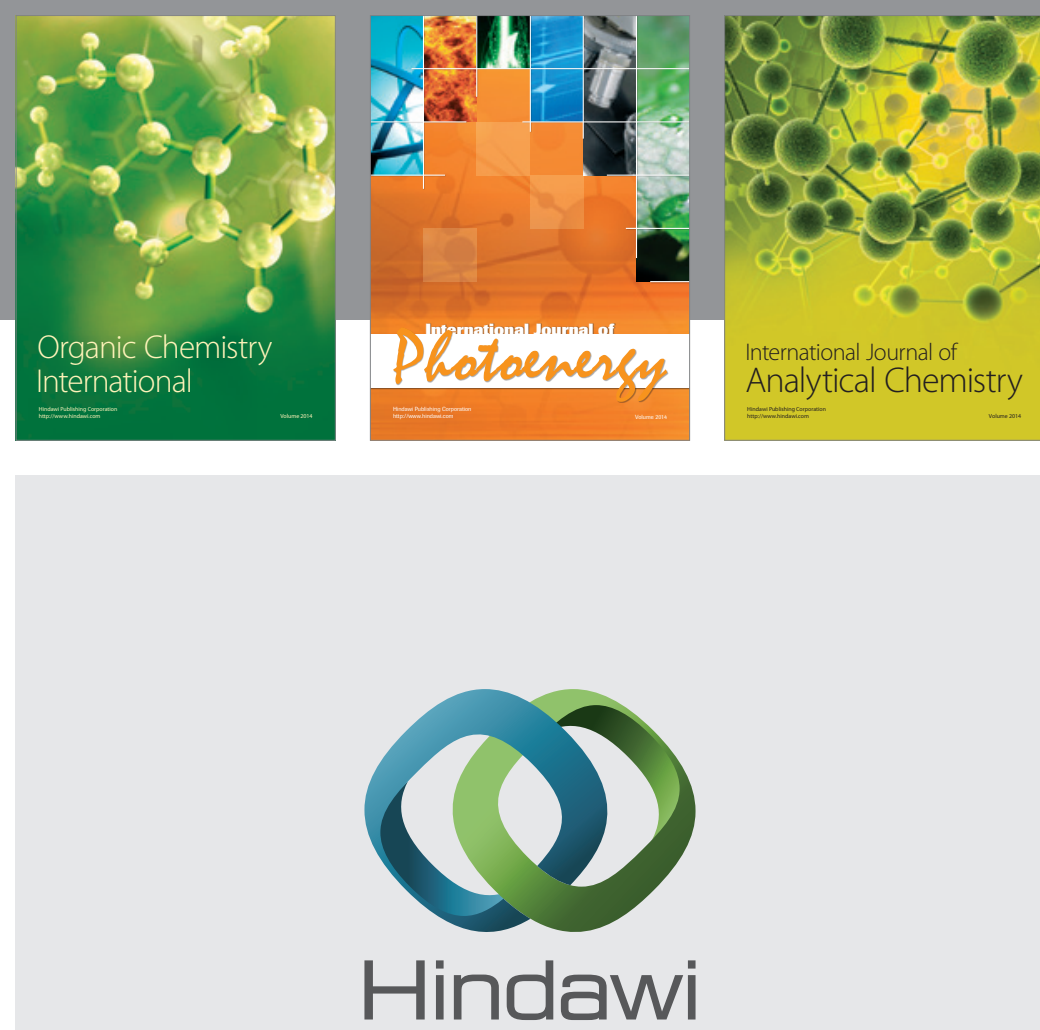

Submit your manuscripts at

http://www.hindawi.com
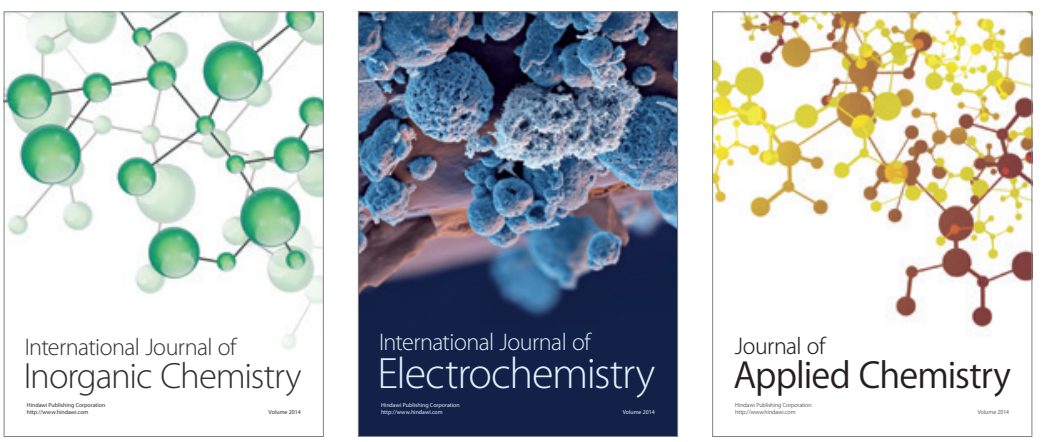

Journal of

Applied Chemistry
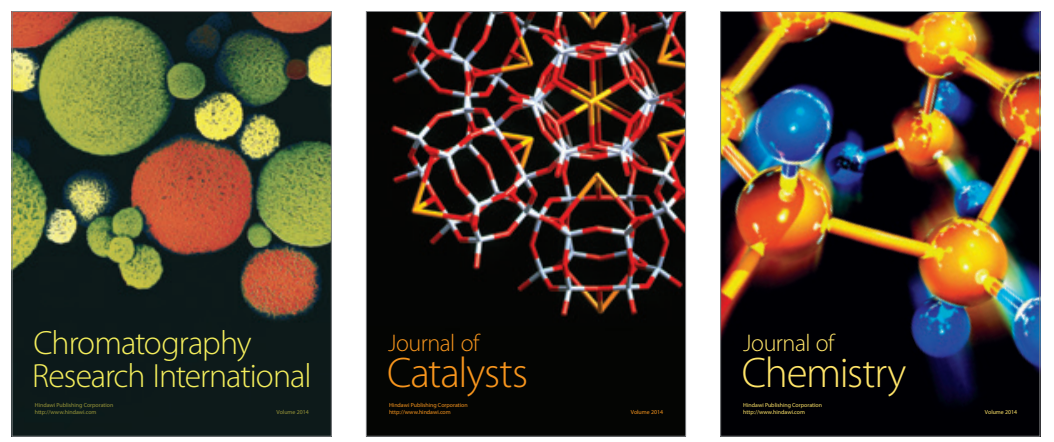
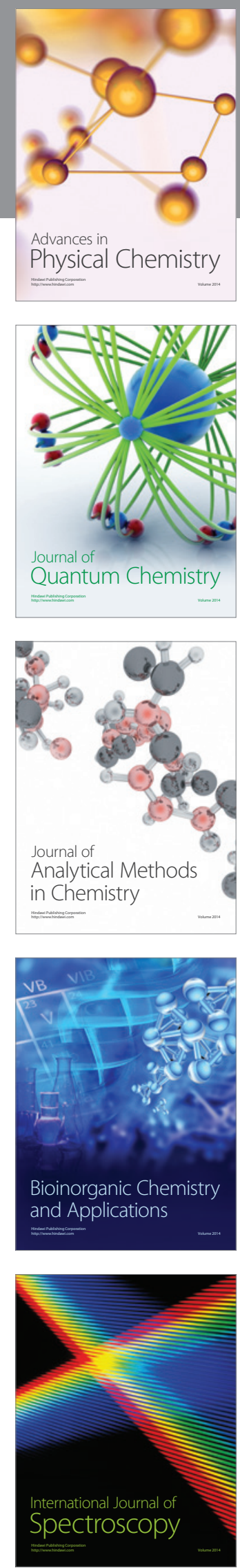